\title{
Postoperative Strahlentherapie beim Mammakarzinom
}

\author{
J. Dunst \\ Klinik für Strahlentherapie, Martin-Luther-Universität Halle-Wittenberg
}

\section{Postoperative Radiotherapie nach brusterhaltender Operation}

Mehr als die Hälfte aller Patientinnen kann heute brusterhaltend behandelt werden. Nach einer brusterhaltenden Operation (Tumorektomie, Segmentektomie) ist die postoperative Bestrahlung der Restbrust obligat. Die Radiotherapie senkt die Rate lokaler Rückfälle in der Restbrust von 30-40\% auf etwa 5-10\%, wobei der Effekt mit zunehmender Nachbeobachtungszeit größer wird [1]. Die Strahlentherapie vergrößert durch bessere lokale Kontrolle also die Chance auf definitiven Erhalt der Brust.

Kontrovers wird bisher diskutiert, ob sich durch die bessere lokale Kontrolle auch ein Überlebensvorteil ergibt. Mehrere Arbeiten der letzten Jahre haben deutlich gezeigt, daß das lokale Rezidiv in der Brust ein wichtiger und eigenständiger prognostischer Faktor ist [2-4]. Wenn also Patientinnen mit einem Lokalrezidiv in der Brust mehr Fernmetastasen entwickeln als lokal kontrollierte Patientinnen, müßte sich dadurch auch die längerfristige Überlebensrate verbessern. Eine Übersicht über die Daten der wichtigsten randomisierten Studien zeigt Tabelle 1. Nur die Studie der NSABP hat eine Nachbeobachtungszeit von mehr als 10 Jahren [5]. In dieser Studie waren die 5-Jahres-Überlebensraten noch nicht signifikant verschieden, jedoch ergibt sich nach 10 Jahren ein signifikanter Vorteil für die postoperativ bestrahlte Gruppe. Die anderen drei Studien zeigen bei Nachbeobachtungszeiten von maximal 5 Jahren keine Unterschiede im Gesamtüberleben [6,7].

Zusammenfassend zeichnet sich ein Überlebensvorteil durch die postoperative Strahlentherapie ab. Die Verhinderung des lokoregionären Rezidivs wirkt also als eine «MetastasenProphylaxe».

\section{Postoperative Strahlentherapie nach Mastektomie}

Im Gegensatz zur Strahlentherapie der Restbrust bei brusterhaltendem Vorgehen wird die postoperative Radiotherapie nach Mastektomie kontrovers diskutiert. Der überwiegende Teil der wissenschaftlichen Daten zur Frage der postoperativen Radiotherapie nach Mastektomie stammt aus Studien, die in den 60er und 70er Jahren durchgeführt wurden und damit zu den ersten randomisierten Therapieprotokollen überhaupt gehören. Man verglich eine postoperative Bestrahlung gegenüber keiner Nachbehandlung. Eine erste Metaanalyse der Daten vor 12 Jahren ergab keinen Überlebensvorteil durch die Anwendung der postoperativen Radiotherapie; ganz im Gegenteil hatten Langzeitüberlebende nach Strahlentherapie ein erhöhtes Sterberisiko im Vergleich zu Patientinnen, die keine Nachbestrahlung erhalten hatten. Aus heutiger Sicht sind die Daten jedoch kritisch zu hinterfragen, und dieser Aspekt wurde in den letzten Jahren in mehreren Arbeiten detailliert abgehandelt. Die Strahlentherapie erfolgte in fast allen Studien mit antiquierten, heute als fehlerhaft anzusehenden Methoden und einer nach heutigem Verständnis zu niedrigen und deshalb ineffektiven Gesamtdosis. Durch ungünstige Bestrahlungstechniken war die Herzbelastung vor allem bei linksseitiger Lokalisation sehr hoch; dies erklärt eine erhöhte kardiale Mortalität bei Langzeitüberlebenden. In einer späteren Metaanalyse ergab sich dann aber eine signifikante Reduktion der Mortalität an Brustkrebs durch die Nachbestrahlung [8]. Eine aktuelle Metaanalyse von 36 Studien mit mehr als 17.000 Patientinnen wurde auch auf dem diesjährigen ASCO-Meeting vorgestellt. Die Daten bestätigen, daß die postoperative Strahlentherapie in den nach 1970 begonnenen und deshalb mit relativ moderner Strahlentherapietechnik durchgeführten Studien eine signifikante Verbesserung des Gesamtüberlebens bewirkt [9].

Viele der genannten Studien sind für heutige Verhältnisse nur bedingt aussagekräftig, weil sie keine adjuvante Chemotherapie oder Hormontherapie (zumindest nicht obligat) enthielten. Heute werden aber fast alle Patientinnen adjuvant behandelt. Es stellt sich daher die Frage, ob ein signifikanter Vorteil durch die Strahlentherapie auch dann resultiert, wenn eine zusätzliche Systemtherapie erfolgt.

Die Daten der beiden größten Studien zu dieser Frage, nämlich die Studie der Danish Breast Cancer Study Group und der British Columbia Trial, wurden kürzlich publiziert [10,11]. In der dänischen Studie wurden Patientinnen nach Mastektomie mit sechs Kursen Cyclophosphamid, Methotrexat, 5-Fluorouracil (CMF) behandelt und hinsichtlich der Nachbestrahlung randomisiert [10]. Die Patientenrekrutierung erfolgte von 1982 bis 1989. Die Strahlentherapie entsprach den heute üblichen

\begin{tabular}{ll}
\hline KARGER & (1) 1999 S. Karger GmbH, Freiburg \\
Fax +49 761 4520714 & Accessible online at: \\
$\begin{array}{l}\text { E-mail kargergmbh@aol.com } \\
\text { www.karger.com }\end{array}$ & http://BioMedNet.com/karger \\
\end{tabular}

Prof. Dr. Jürgen Dunst

Martin-Luther-Universität Halle-Wittenberg Klinik für Strahlentherapie

Dryanderstraße 4-7, D-06097 Halle (Deutschland) Tel. 0345-5574310, Fax -5574333

E-mail juergen.dunst@medizin-uni-halle.de 
Tab. 1. Übersicht über die randomisierten Studien zur postoperativen Radiotherapie nach brusterhaltender Operation. In allen Studien reduziert die Strahlentherapie die lokale Rezidivrate. In der Studie mit der längsten Nachbeobachtungszeit resultiert daraus ein signifikanter Überlebensvorteil. Bei den anderen Studien ist die Nachbeobachtungszeit zu kurz [nach 7]

\begin{tabular}{|c|c|c|c|c|c|c|}
\hline Studie & Einschlußkriterien & Therapie & $\mathrm{N}$ & $\begin{array}{l}\text { Lokal- } \\
\text { rezidive } \\
\%\end{array}$ & $\begin{array}{l}\text { Gesamt- } \\
\text { überleben } \\
\%(p)\end{array}$ & $\begin{array}{l}\text { Follow-up } \\
\text { Jahre }\end{array}$ \\
\hline NSABP-B06 & $\begin{array}{l}\text { Primärtumor }<4 \mathrm{~cm} \\
\text { Stadium I/II }\end{array}$ & $\begin{array}{l}\mathrm{L} \\
\mathrm{L}+\mathrm{RT}\end{array}$ & $\begin{array}{l}520 \\
515\end{array}$ & $\begin{array}{l}32 \\
8^{*}\end{array}$ & $\begin{array}{l}65 \\
71(0,04)\end{array}$ & 10 \\
\hline Kanada & $\begin{array}{l}\text { Primärtumor }<4 \mathrm{~cm} \\
\text { Resektionsrand frei }\end{array}$ & $\begin{array}{l}\mathrm{L} \\
\mathrm{L}+\mathrm{RT}\end{array}$ & $\begin{array}{l}421 \\
416\end{array}$ & $\begin{array}{c}30 \\
8^{*}\end{array}$ & $\begin{array}{l}86 \\
88(0,45)\end{array}$ & 5 \\
\hline $\begin{array}{l}\text { Uppsala- } \\
\text { Ørebro }\end{array}$ & $\begin{array}{l}\text { Primärtumor }<2 \mathrm{~cm} \\
\text { Unifokal, } \\
\text { Alter }<80 \text { Jahre }\end{array}$ & $\begin{array}{l}\mathrm{L} \\
\mathrm{L}+\mathrm{RT}\end{array}$ & $\begin{array}{l}197 \\
184\end{array}$ & $\begin{array}{l}18,4 \\
2,3 * *\end{array}$ & $\begin{array}{l}90 \\
91(0,44)\end{array}$ & 5 \\
\hline Mailand III & $\begin{array}{l}\text { Primärtumor }<2,5 \mathrm{~cm} \\
\text { N0/N1 }\end{array}$ & $\begin{array}{l}\text { Quad } \\
\text { Quad + RT }\end{array}$ & $\begin{array}{l}273 \\
294\end{array}$ & $\begin{array}{l}8,8 * * * \\
0,3\end{array}$ & $\begin{array}{l}96 \\
97(0,85)\end{array}$ & 3,5 \\
\hline
\end{tabular}

$* \mathrm{p}<0,001 ; * * \mathrm{p}<0,0001 ; * * * \mathrm{p}<0,01$.

$\mathrm{L}=$ Lymphektomie; $\mathrm{RT}=$ Radiotherapie Q Quad $=$ Quadrantektomie.
Qualitätskriterien und umfaßte eine Bestrahlung der Brustwand und der regionären Lymphknoten. In der Radiotherapiegruppe war die lokale Rezidivrate signifikant niedriger als in der Gruppe mit CMF allein ohne Radiotherapie (9 vs. $32 \%$ nach 10 Jahren). Dadurch verbesserte die Radiotherapie das krankheitsfreie Überleben nach 10 Jahren von 34 auf $48 \%$ $(\mathrm{p}<0,001)$ und das Gesamtüberleben von 45 auf $54 \%$ $(p<0,001)$. Im British Columbia Trial verbesserte die zusätzliche Strahlentherapie das Gesamtüberleben nach 10 Jahren um 10\% (49\% im Arm mit CMF allein vs. 59\% im Arm mit CMF plus Strahlentherapie). Die Verbesserungen des Gesamtüberlebens in den beiden Studien entsprechen etwa dem Effekt einer adjuvanten Hormon- oder Chemotherapie, die ebenfalls eine Verbesserung der 5- und 10-Jahres-Überlebensraten um knapp $10 \%$ bewirkt [12]. Möglicherweise spielt die Chemotherapie für die Wirksamkeit der Radiotherapie auch in dieser Studie eine Rolle, ähnlich wie es im obengenannten B06-Protokoll vermutet werden muß. Das würde bedeuten, daß Risikopatienten von einer Kombination aus Chemo- und Radiotherapie einen besonders großen Gewinn haben durch eine synergistische Wirkung beider Modalitäten.

Die Daten des British Columbia Trial wurden auf dem diesjährigen ASCO-Meeting erneut vorgestellt [11]. Die zusätzliche Strahlentherapie konnte die Gesamtmortalität signifikant um $30 \%$ senken. Dieser Effekt war auch bei Patientinnen mit relativ günstiger Prognose, nämlich bei Befall von 1-3 axillären Lymphknoten, vorhanden (Tab. 2); er war in dieser Subgruppe sogar am größten. Dieser Befund ist neu, denn bisher wurde der Effekt der Strahlentherapie im Hinblick auf die Verbesserung des Gesamtüberlebens vor allem bei Patientinnen mit ausgedehntem Lymphknotenbefall gefunden.

Ein positiver Einfluß der Strahlentherapie auf das Gesamtüberleben besteht vermutlich auch bei postmenopausalen Patientinnen, die eine adjuvante Hormontherapie erhalten. Die Daten der dänischen Studie zu diesem Thema wurden kürzlich publiziert [13] und sind auszugsweise in Tabelle 3 dargestellt.
Tab. 2. Einfluß der postoperativen Strahlentherapie nach Mastektomie auf die Gesamtüberlebensrate, aktuelle Daten des British Columbia Trial [11] ${ }^{1}$

\begin{tabular}{lrl}
\hline Kollektiv & N & $\begin{array}{l}\text { Reduktion der } \\
\text { Mortalität }\end{array}$ \\
\hline Alle Patientinnen & 318 & 0,70 \\
1-3 positive LK, kein extrakapsulärer Befall & 108 & 0,73 \\
1-3 positive LK, extrakapsulärer Befall & 71 & 0,47 \\
$>4$ positive LK, kein extrakapsulärer Befall & 35 & 1,06 \\
$>4$ positive LK, extrakapsulärer Befall & 75 & 0,71 \\
\hline
\end{tabular}

LK = Lymphknoten .

${ }^{1}$ Angegeben ist das relative Sterberisiko im Strahlentherapiearm im

Vergleich zur Kontrolle, Werte unter 1 bedeuten also einen Überlebensvorteil durch die Strahlentherapie.

\section{Radiotherapie beim lokoregionären Rezidiv}

Patientinnen mit einem lokalen Rezidiv haben im Vergleich zu Patientinnen mit lokaler Tumorkontrolle eine signifikant schlechtere Überlebensrate. Wichtigste Prognosefaktoren bei manifestem Lokalrezidiv sind der initiale axilläre Lymphknotenstatus ( $\mathrm{pN} 0$ besser als $\mathrm{pN}+$ ) und das rezidivfreie Intervall (je länger, desto bessere Prognose; im allgemeinen gilt ein rezidivfreies Intervall von mindestens 2 Jahren als günstig). Bei günstiger Konstellation (pN0 und/oder krankheitsfreies Intervall > 2 Jahre) und Erreichen einer lokalen kompletten Remission durch nochmalige Operation und/oder Radiotherapie überleben mehr als $50 \%$ der Patientinnen mit Lokalrezidiv 5 Jahre. Bei ungünstiger Prognose (initial pN+ und krankheitsfreies Intervall <2 Jahre) liegt die 5-Jahres-Überlebensrate unter $30 \%$, bei unbeherrschtem lokalen Tumor überlebt kaum eine Patientin länger als 3 Jahre. Der Wert einer Systemtherapie, also Chemo- oder Hormontherapie, beim lokalen Rezidiv läßt sich wegen fehlender Daten nicht beurteilen. In den letzten 15 Jahren ist nur eine randomisierte Studie zu dieser Frage publiziert worden. In dieser Studie der SAKK wurden Patientinnen mit lokalem, hormonrezeptorpositivem Rezidiv operiert und nachbestrahlt und dann randomisiert in einen Kontrollarm ohne Systemtherapie oder einen Arm mit adjuvanter Tamoxifenbehandlung [14]. Die Fernmetastasie- 
rungsrate und das Gesamtüberleben wurde durch Tamoxifen nicht beeinflußt, so daß zum jetzigen Zeitpunkt keine Daten existieren, die eine wie auch immer geartete Systemtherapie als Standardbehandlung rechtfertigen würden. (Die Frage der Systemtherapie beim lokoregionären Rezidiv wird zur Zeit in der multizentrischen GBSG-Studie 6 geprüft. Informationen erhältlich über Dr.H.F. Rauschecker, Fax: (08031) 381367.) Demgegenüber gilt als sicher, daß man bei einem lokoregionär begrenzten Rezidiv eine lokale Kontrolle anstreben muß. Aus diesem Grunde sind resektable Rezidive grundsätzlich einer Operation zuzuführen. Patientinnen, die noch nicht bestrahlt worden sind, sollten eine postoperative Radiotherapie erhalten. Falls im Rahmen der Primärbehandlung bereits eine Radiotherapie erfolgte, ist eine nochmalige kleinvolumige Bestrahlung indiziert, wenn die Resektion nicht radikal ist (R1 oder R2) oder Inoperabilität besteht.

\section{Stellung der Radiotherapie im multimodalen Behandlungskonzept des Mammakarzinoms}

Die Strahlentherapie verbessert die lokoregionäre Tumorkontrolle und kann durch die Verhinderung des Lokalrezidivs eine echte «Metastasen-Prophylaxe» darstellen. Die Radiotherapie ist deshalb immer dann indiziert, wenn ein substantielles lokoregionäres Rezidivrisiko besteht. Eine Indikation für eine postoperative Strahlentherapie besteht nach aktuellem Kenntnisstand eindeutig in folgenden Situationen [15]:

1. Nachbestrahlung nach brusterhaltender Operation

- Radiotherapie der Restbrust obligat.

- Bestrahlung der Lymphabflußgebiete bei (ausgedehntem) axillärem Befall.

2. Nachbestrahlung nach Mastektomie

- Bei pT3-4 unabhängig vom Lymphknotenbefall.

- Bei ausgedehntem axillärem Befall (bei $>4$ positiven LK).
Tab. 3. Einfluß der zusätzlichen postoperativen Radiotherapie nach Mastektomie auf das Gesamtüberleben bei postmenopausalen Patientinnen mit adjuvanter Hormontherapie [13]

\begin{tabular}{lccl}
\hline & $\begin{array}{l}\text { Mastektomie } \\
\text { + TAM }\end{array}$ & $\begin{array}{c}\text { Mastektomie } \\
+ \text { TAM + } \\
\text { postoperative } \\
\text { Radiotherapie }\end{array}$ & p \\
& \multicolumn{4}{c}{} \\
\hline Patientenzahl & 689 & 686 & $<0,001$ \\
Lokoregionales Rezidiv, \% & 35 & 8 & $<0,001$ \\
Krankheitsfrei, \% & 24 & 36 & $=0,03$ \\
10-Jahres-Überleben, \% & 36 & 45 & \\
\hline
\end{tabular}

TAM $=$ Tamoxifen

- Bei geringem axillärem Befall (bei 1-3 positiven LK) bei postmenopausalen Patientinnen.

Systemtherapie, also Chemo- bzw. Hormontherapie, und zusätzliche Strahlentherapie schließen sich nicht aus. Ganz im Gegenteil scheint eine Kombination aus Strahlentherapie und Systemtherapie besonders wirksam zu sein. Über die Frage, in welcher zeitlichen Sequenz man die einzelnen Modalitäten am besten staffelt, bestehen zum Teil kontroverse Meinungen. Die Wirksamkeit der Systemtherapie, insbesondere der Chemotherapie, ist um so größer, je früher sie nach der Operation beginnt. Die Radiotherapie sollte ebenfalls möglichst bald postoperativ eingesetzt werden, jedoch ist eine Verschiebung insbesondere bei kurzen oder intensiven Chemotherapien aus Toxizitätsgründen sinnvoll. Aus klinisch-praktischen Gründen empfiehlt sich bei CMF-Therapie das Sandwich-Schema $(3 \times \mathrm{CMF}$-Strahlentherapie $-3 \times \mathrm{CMF})$ und bei anthrazyklinhaltigen Regimen die sequentielle Chemo-Radiotherapie $(4 \times$ AC-Strahlentherapie). Eine Hormontherapie kann zeitgleich zur Strahlentherapie erfolgen.

\section{Literatur}

1 Dunst J: Adjuvante Strahlentherapie beim operablen Mammakarzinom. Onkologe 1995;1:205-213.

2 Fisher B, Anderson S, Fisher ER, Redmond C, Wickerham DL, Wolmark N, Mamoumas EP, Deutsch M, Margolese R: Significance after ipsilateral breast tumor recurrence after lumpectomy. Lancet 1991 338:327-331.

3 Kemperman H, Borger J, Hart A, Peterse H, Bartelink H, van Dongen J: Prognostic factors for survival after breast conserving therapy for stage I and II breast cancer: The role of local recurrence. Eur J Cancer 1995;31A:690-698.

4 Veronesi U, Luini A, Galimberti V, Zurrida S: Conservation approaches for the management of stage I/II carcinoma of the breast: Milan Cancer Institute trials. World J Surg 1994;18:70-75.

5 Fisher B, Anderson S, Redmond CK, Wolmark N, Wickerham DL, Cromin WM: Reanalysis and results after 12 years follow-up in a randomized trial comparing total mastectomy with lumpectomy with or without irradiation in the treatment of breast cancer. N Engl J Med 1995;333:1456-1461.

6 Levitt SH, Aeppli DM, Nierengarten ME: The importance of local control in the conservative treatment of breast cancer. Acta Oncol 1995;34:839-844.
7 Sauer R: Einfluß der lokalen Kontrolle auf das Gesamtüberleben nach brusterhaltender Therapie des Mammakarzinoms. Strahlenther Onkol 1996; 172:181-185.

8 Cuzick J, Stewart H, Rutquist L, Houghton J, Edwards R, Redmond C, Peto R, Baum M, Fisher B, Host $\mathrm{H}$ : Cause-specific mortality in long-term survivors of breast cancer who participated in trials of radiotherapy. J Clin Oncol 1994;12:447-453.

9 Van de Steene J, Storme G: Meta-analysis of breast cancer surgery trials confirms significant factors for overall survival benefit due to adjuvant radiotherapy. ASCO Proc 1999.

10 Overgaard M, Hansen PS, Overgaard J, Rose C, Andersson M, Bach F, Kjaer M, Gadeberg CC, Mouridsen HT, Jensen MB, Zedeler K: Postoperative radiotherapy in high-risk premenopausal women with breast cancer who receive adjuvant radiotherapy: Danish Cancer Cooperative Group 82b Trial. N Engl J Med 1997;337:949-955.

11 Ragaz J, Jackson S, Le N, Manji M, Wilson K, Olivotto I, Coldman A, Spinelli J: Postmastectomy radiation $(\mathrm{RT})$ outcome in node $(\mathrm{N})$ positive breast cancer patients among N1-3 versus N4+ subset: Impact of extracapsular spread (ES). Update of the British Columbia randomized trial. ASCO Proc 1999.
12 Early Breast Cancer Trialists' Collaborative Group: Systemic treatment of early breast cancer by hormonal, cytotoxic, or immune therapy. Lancet 1992; 339:1-15.

13 Overgaard M, Jensen MB, Overgaard J, Hansen PS, Rose C, Andersson M, Kamby C, Kjaer M, Gadeberg CC, Rasmussen BB, Blichert-Toft M, Mouridsen HT: Postoperative radiotherapy in highrisk postmenopausal breast-cancer patients given adjuvant tamoxifen: Danish Cancer Cooperative Group DBCG 82c randomised trial. Lancet 1998; 353:1641-1648.

14 Borner M. Bacchi M, Goldhirsch A, Greiner R, Harder F, Castiglione M, Jungi MF, Thurlimann B, Cavalli F, Obrecht JP: First isolated locoregional recurrence following mastectomy for breast cancer: Results of a phase-III multicenter study comparing systemic treatment with observation after excision and radiation. J Clin Oncol 1994;12:2071-2077.

15 Hortobagyi GN:Treatment of breast cancer. N Engl J Med 1998;339:974-984.

16 Whelan T, Clark R, Roberts R, Levine M, Foster G: Ipsilateral breast tumor recurrence postlumpectomy is predictive of subsequent mortality: results from a randomized trial. Int $\mathbf{J}$ Radiat Oncol Biol Phys 1994;30:11-16. 